\title{
Attitude Output of Strapdown Inertial Navigation System Based on Laser Gyro
}

\author{
Jianzhong Wang, \\ College of Artificial Intelligence, Chongqing Youth Vocational and Technical College, Chongqing, \\ 400712, China \\ Yan Zhang \\ Department of Economics Management, Chongqing Youth Vocational and Technical College, \\ Chongqing, 400712, China. \\ Jijun Yan \\ Department of Economics Management, Chongqing Youth Vocational and Technical College, \\ Chongqing, 400712, China.
}

Received: January 1. Revised: August 25, 2020. 2nd Revised: November 20, 2020. Accepted: November 23, 2020.

Published: November 24, 2020

\begin{abstract}
Aiming at the real-time problems of signal acquisition, attitude calculation and data exchange of strapdown inertial navigation system, the data exchange between the core device of three-axis screw instrument and three-axis accelerometer sensor inertial unit (IMU) is analyzed. The RS-232 serial interface and can bus interface are adopted, which can not meet the requirements of high-speed sampling and real-time data transmission of each sensor. A new method based on FPGA dual port RAM and dual DSP is proposed Speed data access mode, through the main control CPU clock synchronization, can effectively solve the bottleneck problem of data communication between IMU attitude data and core equipment, and realize the rapid response ability of vehicle navigation system. Experiments and simulations show that the highest frequency attitude update rate of the method can reach $2000 \mathrm{kHz}$, which can effectively solve the input and output data and navigation calculation ability, and improve the maneuverability of the carrier.
\end{abstract}

Keywords - Inertial Measurement Unit; Field Programmable Gate Array; Dual Port Random Access Memory; Accelerometer; Laser Gyro;

\section{INTRODUCTION}

$\mathrm{C}_{\text {TRAPDOWN inertial navigation system (SINS) provides }}$ Scarrier position coordinates, attitude, speed, mileage and other information. It has good concealment, working environment is not easy to be disturbed by the outside world, and is not easy to be restricted by meteorological conditions. It is an autonomous, concealment, real-time and all-weather navigation system. The system is composed of inertial measurement unit (IMU) composed of three-axis gyroscope and three-axis accelerometer, odometer, altimeter, Beidou / GPS, etc. . Data acquisition, input, calculation and output is a complex process, involving many sensors, and multiple calculation processing signals are not synchronized, calculation results, output, etc. cause delay, especially in the process of motion Therefore, it is necessary to study the real-time attitude of strapdown inertial navigation system [1].

At present, with the continuous progress of information technology, the development trend of miniaturization and miniaturization of strapdown inertial navigation system is speeding up. The core devices are mainly gyroscope sensors and acceleration sensors. The gyroscopes mainly include liquid floated gyroscope, air floated gyroscope, electrostatic gyroscope, disturbing gyroscope, laser gyroscope, etc. the accelerometers mainly include quartz flexible accelerometer, accelerometer, MEMS accelerometer, piezoelectric accelerometer, etc[2]. In strapdown navigation system, gyroscope and acceleration three axes are orthogonal each other. The linear motion information and angular motion information of the carrier can be measured respectively. Then, the acceleration and angular velocity are transmitted to the navigation computer, and then the error is compensated. Then, the initial attitude matrix is calculated by using the initial accelerometer information, and then the compensated accelerometer along the carrier coordinate system is compensated by the attitude matrix The specific force component is transformed into the accelerometer specific force component along the navigation coordinate system[3]. The velocity and position information are obtained by using the acceleration force component, initial velocity and initial position information. The intermediate results of navigation calculation are fed back to the attitude 
matrix, and the attitude matrix is updated with the compensated gyroscope information. At the same time, the attitude matrix is calculated to obtain the attitude angle [4].

The data acquisition and update rate of gyroscope and accelerometer is an important index of attitude tracking system. High precision strapdown inertial navigation system abroad is mainly developed in the United States and Europe, mainly represented by Litton, Honeywell, AGEMA and other companies[5]. Its attitude accuracy can reach 0.01 degree and its attitude update rate can reach more than $1000 \mathrm{khz}$. The core components of strapdown inertial navigation system are optical gyroscope, mechanical gyroscope and other sensors. The main production units are the 707 Research Institute of China Shipbuilding Corporation, the 618 Research Institute of China aerospace industry, the 13th Research Institute of the Ninth Research Institute of China Aerospace Science and Technology Group Corporation. The data acquisition and calculation computer design of strapdown inertial navigation system is simple, and the single CPU calculation is not high The parallel processing ability of multi CPU can not synchronize effectively in real time, and can't complete the whole cycle sampling and calculation in a short period of time. At present, the data of gyroscopes and accelerometers are usually sampled by single CPU, and the data receiving and sending mode of each processing unit generally adopts RS-232 serial interface to receive and receive data. The highest input and output speed is $115200 \mathrm{bps}$. The carrier's attitude change and DAC frequency are much higher than those transmitted by RS-232 serial interface. The other uses CAN bus speed, the highest transmission rate can reach $1 \mathrm{Mbps}$, and the data is received by interrupt trigger. After the solution is completed, the data is sent after the line state is idle[6]. The above two data exchange methods are difficult to solve the problem of fast calculation and real-time attitude input and output data, can not receive and send multiple sensor data in millisecond level, and can not achieve rapid acquisition of carrier attitude change data by strapdown inertial navigation system.

In this paper, a multi CPU parallel processing method is proposed, which uses dual port RAM data transmission to realize high-speed data acquisition, input, calculation and output . The highest output frequency attitude rate can reach $2000 \mathrm{kHz}$. A clock synchronization signal is used to synchronize with the vehicle system clock, so as to ensure that the attitude of the strapdown inertial navigation system is synchronized with the clock of the vehicle system; the data transmission time of each component of the strapdown inertial navigation system is reduced; the high-speed data access mode of FPGA dual port RAM and dual DSP is used for input and output [7], so as to solve the difficulty of high-speed real-time data receiving and sending of strapdown inertial navigation system, and improve the attitude Dynamic adaptability of state system.

\section{SYSTEM SIGNAL PROCESSING}

\section{A. Working principle of the system}

The inertial measurement unit (IMU) is composed of three-axis gyroscope and three-axis accelerometer core components. The inertial measurement unit (IMU) is bundled and installed parallel to the central axis of the carrier. The laser gyro is sensitive to the angular rate vector of the carrier coordinate system relative to the inertial coordinate system $\bar{\omega}_{i b}^{b}$, The specific force vector of the accelerometer relative to the sensitive carrier coordinate system $\bar{f}_{i b}^{b}$, In order to eliminate the influence of mechanical vibration or sensor jitter on IMU components during the motion of the carrier, it is necessary to first compensate the output data of the core sensor three-axis gyroscope and three-axis accelerometer according to the error model, such as scale factor, bias, installation error, temperature drift, etc., in order to obtain more accurate angular rate $\bar{\omega}_{i b}^{b}$ and specific force $\bar{f}_{i b}^{b}$ of the carrier relative to the inertial system. In order to obtain the attitude information such as course, roll and pitch in strapdown inertial navigation, the mathematical model is used to represent the "mathematical platform" which has the same function as the platform. The mathematical platform is a theoretical platform. In the inertial space, there is an angle $\alpha$ between the carrier coordinate and the navigation coordinate system. The coordinate systems are represented by the transformation matrix. The gyro stability loop constantly updates the attitude matrix by continuously measuring the angular motion of the carrier relative to the inertial space. The heading angle $\phi$, roll angle $\theta$ and pitch $\gamma$ angle $\mathrm{u}$ are calculated for each period. The accelerometer accumulates the $\mathrm{AD}$ sampling value of each period through the integral circuit to obtain the difference value, and the specific force calculation is to project the specific force in the carrier coordinate system to the navigation coordinate system through coordinate transformation, and calculate the velocity and position data in each direction of northeast sky [8]. 


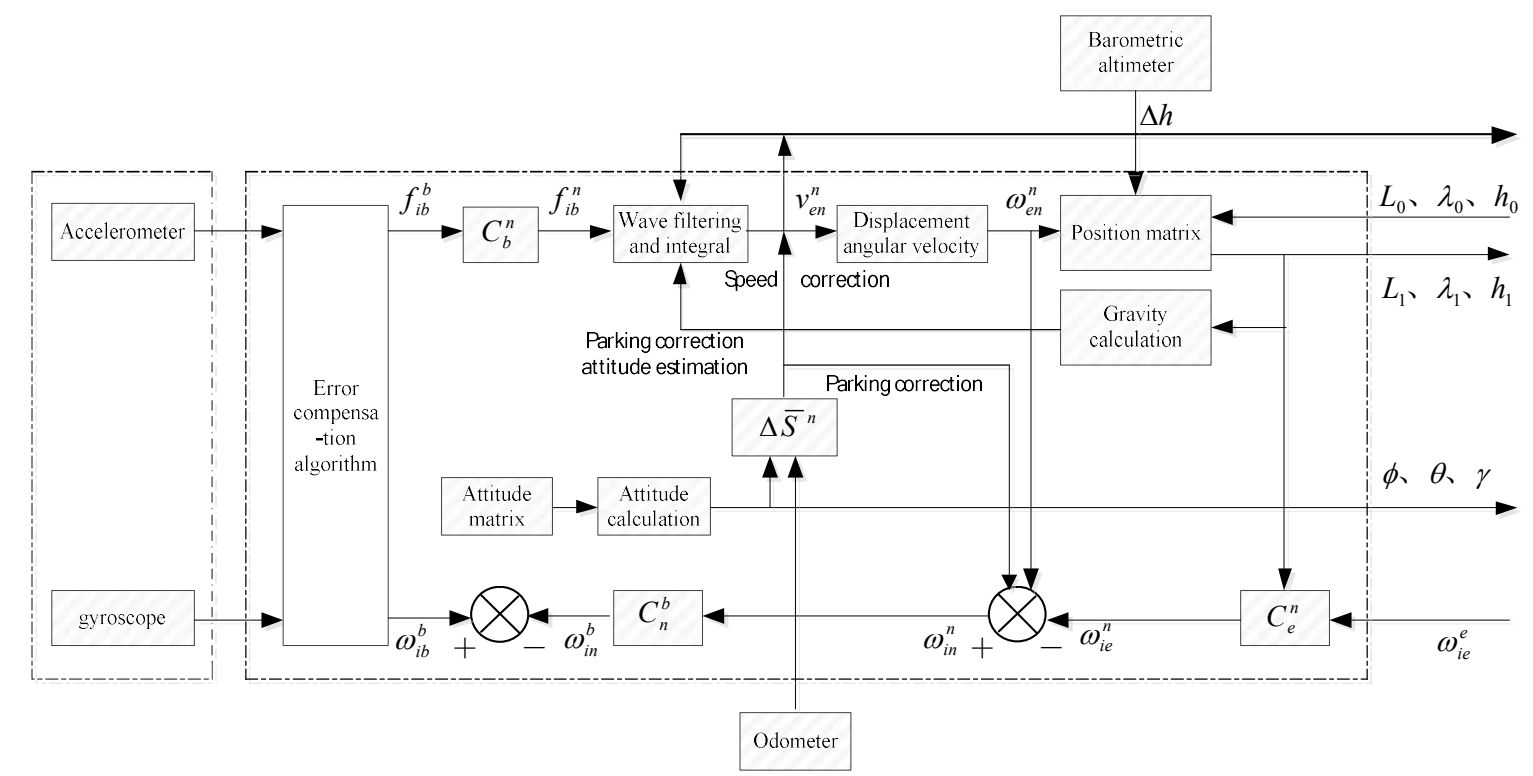

Fig 1. Schematic diagram of strapdown inertial navigation system

\section{B. Signal processing}

TMS320F28335, a 32-bit floating-point digital signal processor, is used for signal processing of three-axis gyroscope and three-axis accelerometer. The chip has high precision, low cost and low power consumption. The operation is as high as $150 \mathrm{MHz}$. There are 176 pins arranged in four groups. The interface is rich, and it can support 3-way serial interface RS-232 at the same time, respectively connect the odometer, altimeter and GPS/Beidou, and set the baud rate $9600 \mathrm{bit} / \mathrm{s}$, data bit 8-bit, even check bit 1 bit and stop bit 1 bit by default. 2-way can bus, respectively output sensor detection data and three-axis gyroscope angular velocity data. Three timers and multiple interrupts, the sampling period is $2000 \mathrm{~Hz}$, which is generated by timer 1 of TMS320F28335 signal processor. The timing period of other collaborative calculation and signal processing CPU is consistent to ensure clock synchronization. FPGA adopts integrated large-scale digital circuit Actel chipset a3p250, which provides $4 \mathrm{I} / \mathrm{O}$ groups, 157 interfaces, 6 clocks and 1024 bit flash memory to effectively improve data access efficiency. Ad7608 is used in a / D converter, which can support 18 bit and 8-channel synchronous analog-to-digital sampling, and the sampling rate of the whole channel is up to $200 \mathrm{ksps}$.

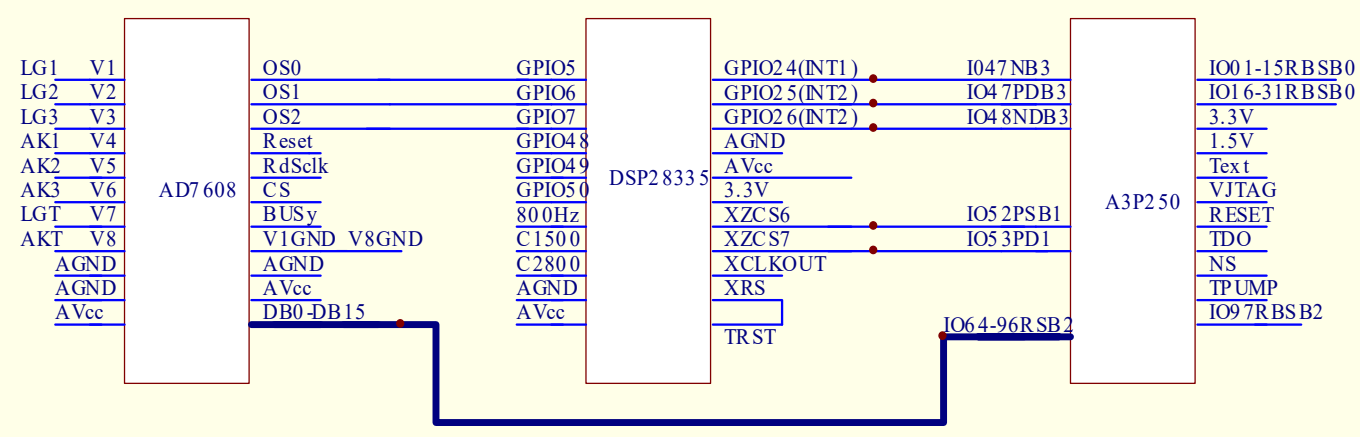

Fig 2. Schematic diagram of signal processing

\section{Data transmission between components}

The signal processing of strapdown navigation system is mainly composed of inertial measurement unit (IMU), odometer, barometric altimeter and GPS/BeiDou module. The data exchange between odometer, altimeter and GPS/BeiDou module is carried out through RS-232 serial interface. The corresponding baud rate, parity check and 1-bit stop bit are set. The external DC power supply of the system is dc $26 \pm 4 \mathrm{~V}$. The odometer, altimeter, GPS/BeiDou and other power supplies are connected from the specified socket of IMU module, and the voltage is $24 \mathrm{~V} \mathrm{DC}$. The gyroscope, accelerometer, circuit board, etc. of IMU components are installed in the box and bundled on the chassis of the car body. The data interaction is realized through the CAN bus input and output with the user control system and IMU, as shown in Figure 3 below: 


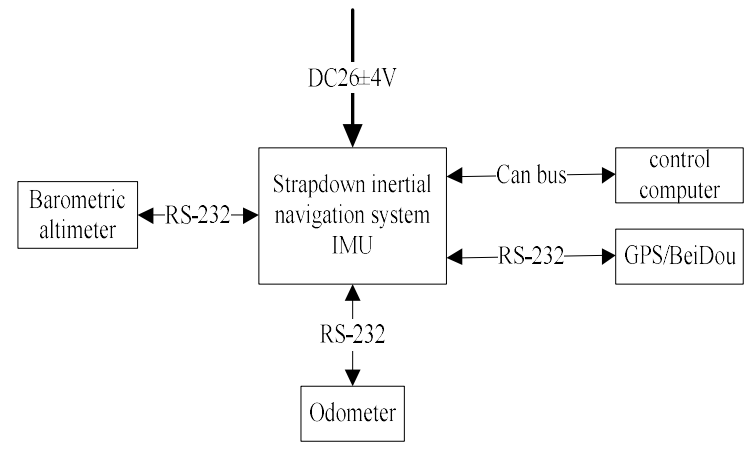

Fig 3. System component data exchange

\section{Navigation computer design}

The navigation computer CPU adopts tms320c6747 chip, 32-bit floating-point operation, main frequency of $300 \mathrm{~Hz}$, up to 24 million floating-point instructions and 18 million floating-point instructions per second, $128 \mathrm{~K}$ memory and two-level cache, 32-bit EMIF, 32-bit flash, FPGA, SDRAM and SRAM, rprom, 3-channel serial interface RS-232 and 2-way I2C bus.

The three serial interfaces of the signal processing board TMS320F28335 are respectively connected with the range meter, altimeter and GPS/BeiDou. One can bus outputs the sensor detection data, the other one outputs the angular velocity of three-axis gyroscope, and the timer outputs $2000 \mathrm{~Hz}$ high-low level, which triggers the interrupt and keeps synchronization with the navigation signal processor tms320c6747. Interrupt 1 frequency is consistent with the system sampling frequency of $2000 \mathrm{~Hz}$ to ensure that ad7608 three-axis gyroscope and three-axis acceleration data are read at the same time; interrupt 2 reads attitude heading angle, roll angle and pitch angle data of Actel chipset a3p250 at $400 \mathrm{~Hz}$; interrupt 3 reads position, speed and range data at $10 \mathrm{~Hz}$.

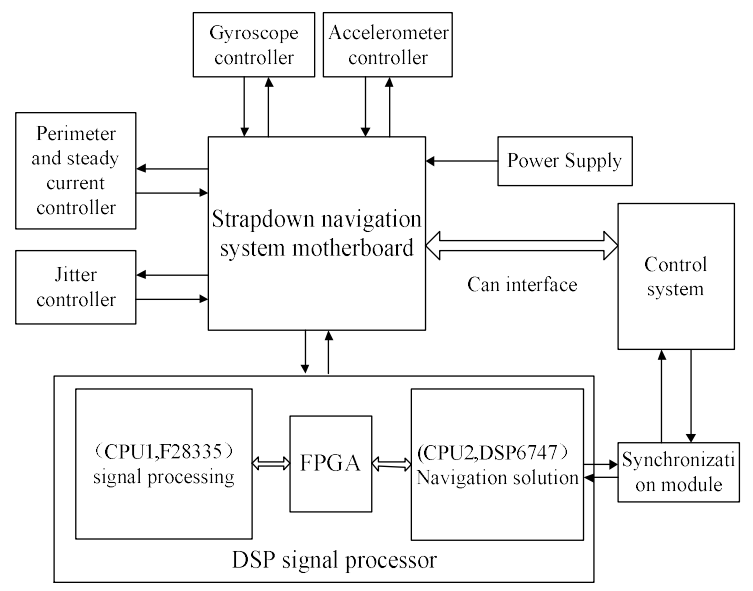

Fig 4. Navigation data block diagram

\section{SENSOR SAMPLING AND ANALYSIS}

\section{A. Gyroscope signal processing analysis}

The core component sensor of strapdown inertial navigation system adopts laser gyroscope. In 1911, Sagnac effect invented a kind of ring interferometer that can rotate [9]. The signal processing circuit can realize the design of phase discrimination, frequency doubling, counting and synchronous control through the output of sensor signal sin and COS signal, so as to measure the frequency difference between positive and negative laser beams in the resonant cavity The angle of the carrier relative to the inertial space.

$$
F=4 A \omega / L \lambda
$$

Where: $\mathrm{F}$ is the frequency difference of two laser beams, a is the area surrounded by the laser loop, $\omega$ is the external input angular velocity, 1 is the cavity length, and $\lambda$ is the optical wavelength.

The period of frequency difference between forward and backward laser waves is as follows:

$$
\begin{array}{r}
N=\int_{0}^{t} f d t=\frac{4 A}{\lambda L} \int_{0}^{t} \Omega d t=\frac{4 A}{\lambda L} \theta \\
\theta=l \lambda N / 4 A
\end{array}
$$

Where: $\theta$ is the angle within $\mathrm{t}$ in a period, $\mathrm{n}$ is the number of interference fringes detected by photodetector in time $\mathrm{t}$. Let $\mathrm{K}$ be the scale factor of the laser gyro:

$$
K=l \lambda / 4 A
$$

The laser gyro cavity is machined with high precision by ultrasonic vibration grinding and compound polishing technology. The rays projected on the cathode by the light source have a small discrete angle $\varepsilon$, and the interference image is formed at the same time:

$$
I=I_{0}\left[1+\operatorname{CoS}\left(2 \pi \varepsilon \chi / \lambda_{n}+f_{0} \varphi_{0}\right)\right]
$$

Where: $\varphi_{0}$ is the fixed phase shift, $\varepsilon=2 n_{1} \theta$ and $n_{1}$ are the refractive index of the prism, and $\theta$ is the error angle of the prism top angle deviating from 90 degrees. When $F_{0}=0$, the interference image remains unchanged. When the ring laser rotates, the interference image moves on the cathode, and its speed depends on the beat frequency. Two photoelectric sensitive units with a distance of $\lambda / 4$, whose size is much smaller than the interference fringe spacing, are used to output beat frequency sine cosine signals with phase difference of 90 degrees . between the two photoelectric sensitive units: 


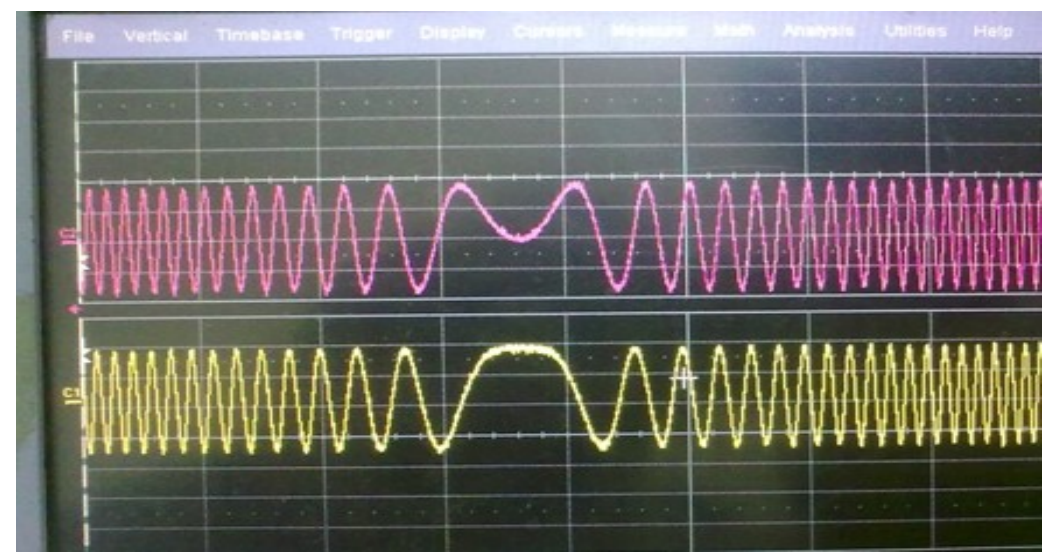

Fig 5. Waveform of output $\sin$ and COS signals

Without considering the bias, the angular rate $\Omega_{r}$ of mechanically dithered RLG includes the external input angular rate $\Omega_{r}$ and jitter $\Omega_{r}$. Therefore, it can be expressed as follows:

$$
\Omega_{r}=\Omega_{i n}+\Omega_{d} \sin \left(\omega_{d} t\right)
$$

Substituting formula (6) into formula (2), we can get the following results:

$$
N=k \int_{0}^{t} \Omega_{i n} d t+k \int_{0}^{t} \Omega_{d} \sin \left(\omega_{d} t\right) d t
$$

When $t=\Delta T=2 \pi / \omega_{d}$ :

$$
k \int_{0}^{t} \Omega_{d} \sin \left(\omega_{d} t\right) d t=0
$$

Therefore, when rounding the period:

$$
\Omega_{r}=\Omega_{\text {in }}
$$

The angular rate input frequency of strapdown inertial navigation system usually ranges from $0 \mathrm{~Hz}$ to $100 \mathrm{~Hz}$, and the jitter frequency of laser gyro is about $400 \mathrm{~Hz}$. It is easy to produce resonance when three gyroscopes are fixed together. Therefore, the three gyroscopes should avoid the same frequency band as far as possible. Generally, digital low-pass filter is designed in engineering application, which can effectively remove jitter bias signal and get the signal to be measured. At the same time, it can eliminate other high-frequency noise and improve the detection accuracy of laser gyro. In order to improve the real-time performance of the system, considering the hardware characteristics of the signal acquisition board CPU and navigation solution CPU, according to the sampling theorem, the digital sampling frequency should be at least twice the maximum jitter frequency of the three laser gyroscopes. Therefore, the sampling frequency of $2 \mathrm{KHz}$ in this scheme can realize high-speed sampling.

\section{B. Perimeter steady current control analysis}

The laser cavity length of the laser gyro must be stable in order to stabilize the natural frequency of the resonator. Due to the influence of temperature drift and structural stiffness, the length of the laser cavity will be changed. The frequency stability of the system can be achieved by designing a frequency stabilization controller. The design of frequency stabilization controller uses single chip microcomputer to control D / a converter max 526 to generate $0-5 \mathrm{~V}$ continuous voltage with a certain step size (such as $20 \mathrm{mV}$ ); the $0-5 \mathrm{~V}$ input voltage is amplified 40 times by high-voltage amplifier, and then becomes $0-200 \mathrm{v}$ continuous adjustable voltage, which is added to the piezoelectric crystal. At the same time, the 12 bit a / D converter is controlled by the single chip microcomputer to obtain the radiation value of the output light intensity of the laser gyro, and then the maximum value of the output light intensity obtained by the mode scanning system is compared, Then, the control signal is driven by D / A to adjust the change of the cavity length of the laser gyro, so that the light intensity output of the laser gyro reaches the maximum value, and then the frequency is stabilized .

\section{Jitter control analysis}

In order to overcome the locking dead zone of laser gyro, dithering control is applied to the laser gyro. When the external angular velocity does not exceed a certain threshold, the output will be zero. Therefore, dither bias is used to eliminate the lock-in effect, but at the same time, the error caused by jitter noise will be brought into gyro output pulse, so jitter data must be deducted by dither modulation and demodulation method. Because the frequency of reference signal of laser gyroscope is relatively fixed, the jitter frequency is generally lower than the sampling frequency. Therefore, the sampling frequency is more than 2 times higher than the jitter frequency of three gyroscopes. The sampling frequency in this paper is $2000 \mathrm{kHz}$. Then the corresponding sampling data are processed by digital filtering to eliminate the influence of mechanical jitter on the three gyroscopes, and at the same time, the noise interference can be suppressed. 


\section{Accelerometer signal processing analysis}

Accelerometer is also the core sensor of strapdown inertial navigation, which is composed of detection mass, support, potentiometer, spring, damper and shell. The spring deformation is determined by detecting the change of relative motion state of mass, and then the acceleration is determined. The potentiometers convert the acceleration into electrical signals. The accelerometer is actually a one degree of freedom oscillation system, so damper must be used to improve the dynamic quality of the system. The working principle of accelerometers can be divided into the following categories: closed loop liquid floated pendulum, torsion pendulum, vibrating string and pendulum integrated gyroscope. According to the number of input axes, triaxial accelerometers can be divided into single axis, double axis and three axis accelerometers. Accelerometer has the advantages of excellent bias stability, good environmental performance, low cost, low voltage analog output, over-current protection, lcc48, integrated temperature sensor and so on.

Quartz flexible accelerometer uses elastic material to support the accelerometer of pendulum assembly. Due to the flexible support technology, the inherent friction torque of bearing support is eliminated. Within the allowable range of measurement accuracy, considering the adverse conditions such as impact, vibration and overload, the combined stiffness of the two flexible rods is reasonably selected to make the elastic moment generated by the two flexible rods small enough, so that the deflection value and scale factor have good long-term stability. Quartz flexible accelerometer has the advantages of simple structure, small volume, high precision and wide temperature range [7]. It is widely used in transportation vehicles, aerospace, mechanical characteristics detection, vehicle condition monitoring and other fields. The composition principle is shown in the figure:

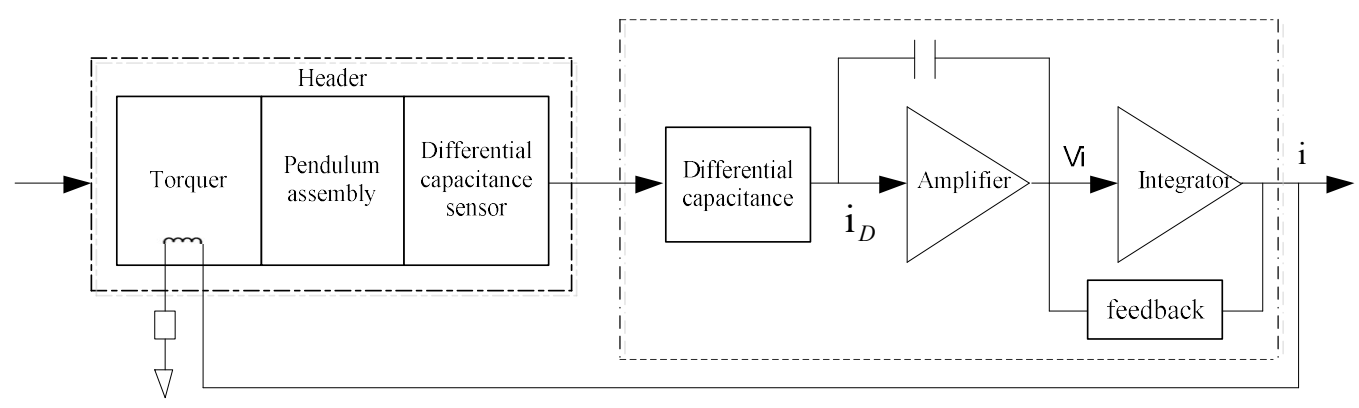

Fig 6. Composition block diagram of accelerometer system

Accelerometers are generally voltage type and current type, and voltage type is adopted in this paper. When there is acceleration input in the process of motion, the pendulum assembly generates the swing moment $M_{g}$ and rotates around the output shaft.

$$
M_{g}=m a L
$$

Where $\mathrm{L}$ is the length of the pendulum.

Differential capacitance sensor produces capacitance difference:

$$
\nabla_{c}=k_{p} \theta
$$

Where: $k_{p}$ is the transfer coefficient of the differential capacitance sensor near the zero position, and $\theta$ is the deflection angle of the pendulum assembly around the output shaft relative to the shell.

The differential capacitance detector is converted into a current signal i:

$$
i=k_{a} \nabla_{c}
$$

The output torque is proportional to the corresponding current:

$$
\mathrm{M}_{t}=\mathrm{k}_{t} i
$$

Where: $\mathrm{k}_{t}$ is a constant, which is the torque coefficient produced by the torquer in the quartz flexible accelerometer.

Under the condition of moment balance, $M_{t}=M_{g}$, then:

$$
I=\left(m l / k_{t}\right) a_{i}
$$

Where: $m l / k_{t}$ current scale factor, the unit when the accelerometer is $1 \mathrm{~m} / \mathrm{s}^{2}$.

The output of quartz flexible accelerometer sensor is through integral resistance and integral capacitance. The voltage value of integral capacitance is read from ad every cycle, and the acceleration value can be obtained by data conversion. 


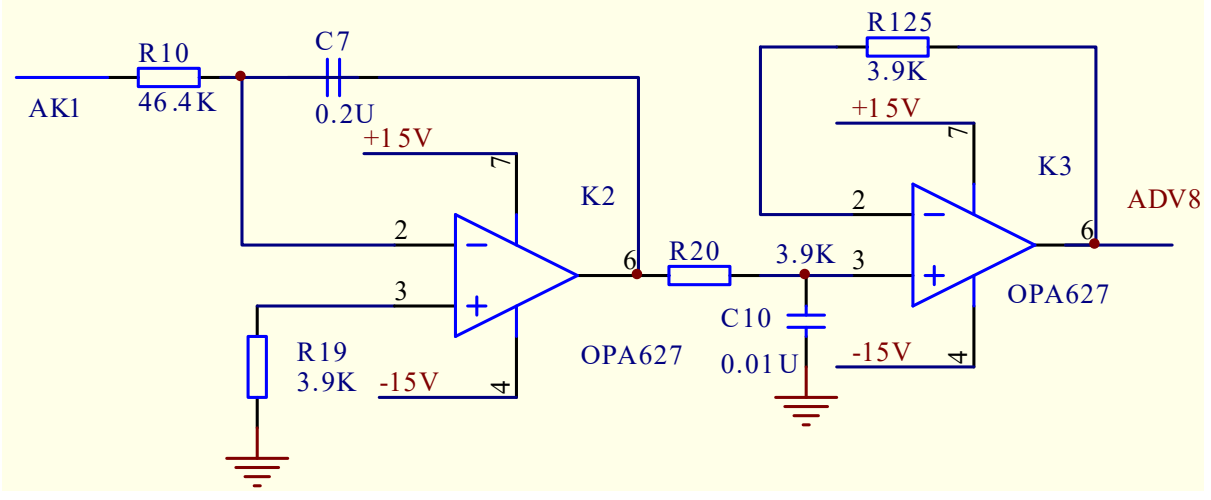

Fig 7. Integration circuit of quartz accelerometer

The accelerometer is fixed on the vibration platform and remains static. The output voltage amplitude of the accelerometer is $5 \mathrm{~V}$. Set the parameter frequency frequency $30 \mathrm{~Hz}$, intensity $20 \%$, maintain the original position vibration, output signal is shown in the figure below:

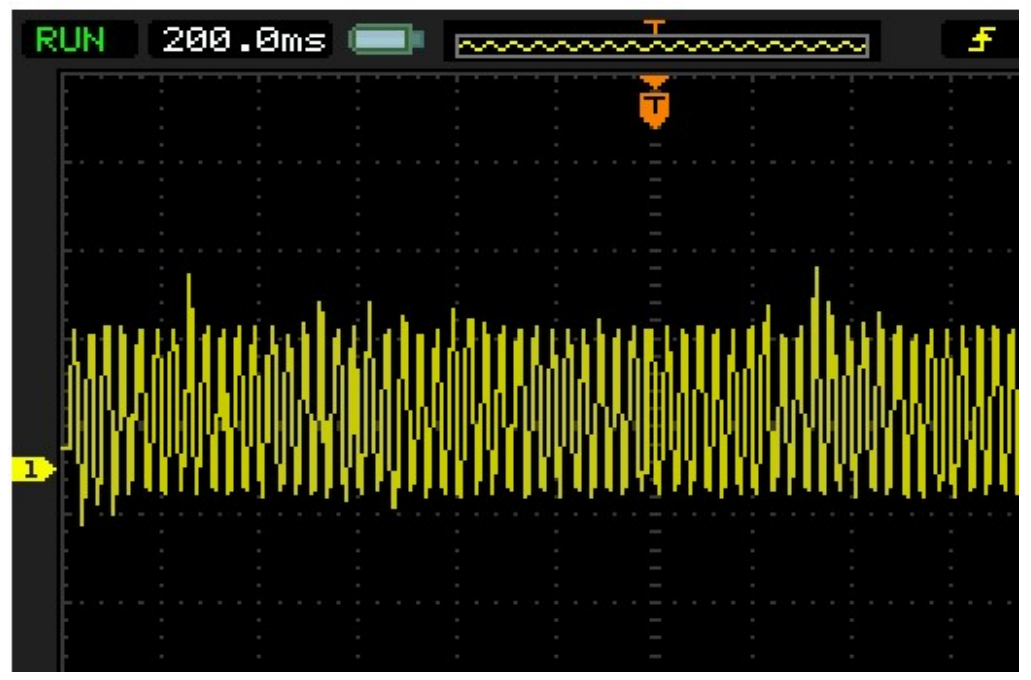

Fig 8. Block diagram of accelerometer system

In this paper, the quartz flexible accelerometer is used to output the current signal which is proportional to the input acceleration. The accelerometer acquisition unit converts the current signal from the accelerometer to the voltage signal through the sampling resistance. After filtering by the analog circuit, the current signal is sent to a / D converter through the differential amplifier and converted into digital signal.

This paper estimates the power spectral density of the sensor output signal after the mechanical vibration reduction by MATLAB, and analyzes its frequency characteristics under the mechanical vibration reduction measures. Due to the limited space, only one vibration experiment is used to illustrate, and the vertical vibration of the simulated shaking table is selected.

(1)In the absence of vibration, the frequency spectrum of the sensor output signal after mechanical vibration reduction is analyzed. After mechanical vibration reduction, the output signal spectrum of accelerometer is basically consistent with that without vibration. The spectrum is shown in the figure: 


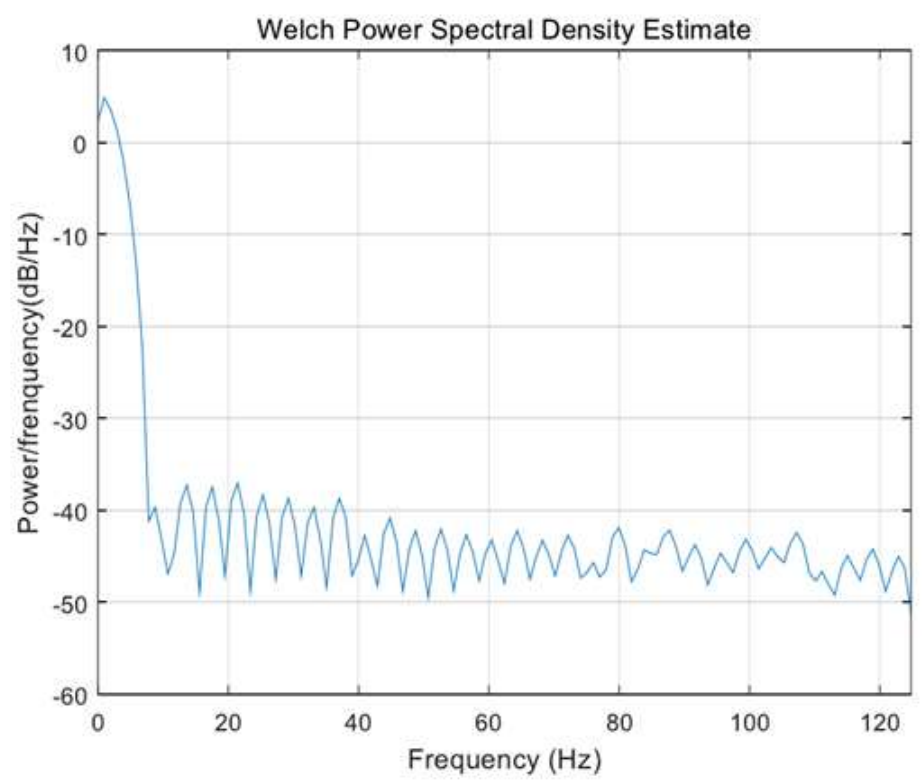

Fig 9. Estimation of vibration without frequency

(2)In the vertical mode, the fixed strength of $30 \%$ and frequency of $50 \mathrm{~Hz}$ is used to analyze the frequency spectrum and the influence of vibration frequency on the output signal of of the sensor output signal after mechanical vibration damping, accelerometer is reduced. The spectrum is shown in the figure:

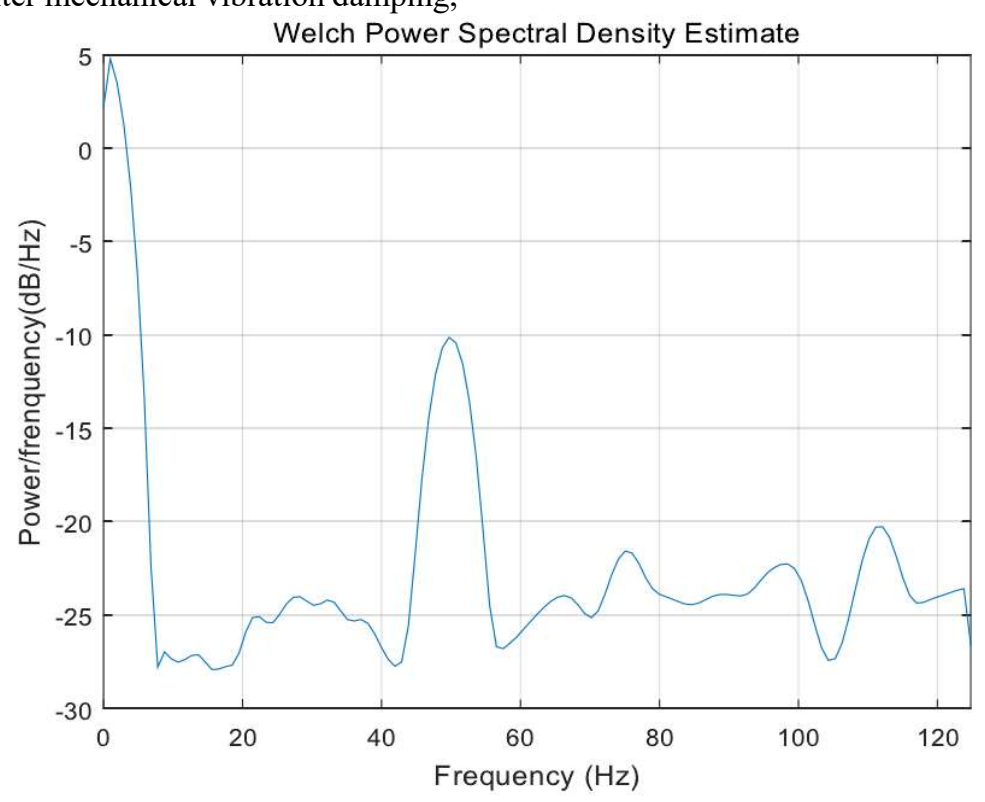

Fig 10. Estimation of vertical vibration frequency

\section{ATTITUDE DATA DELAY AND ANALYSIS}

\section{A. Filter delay analysis}

In order to reduce the impact of RLG jitter, the filter parameter threshold is set for attitude data to ensure the real-time and accuracy of navigation system. Digital filters are divided into finite impulse response FIR and infinite impulse response IIR [11]. In this paper, FIR filter is used, which has the advantages of linear distribution and fixed phase, variable amplitude; the unit sampling response is limited, and the filter performance is relatively stable; the signal is filtered by fast Fourier transform (FFT) algorithm, which can improve the operation accuracy. Disadvantages: digital filtering and Fourier transform have a large amount of calculation work, which requires high system operation ability and hardware conditions, and filtering is easy to cause certain attitude change delay. 


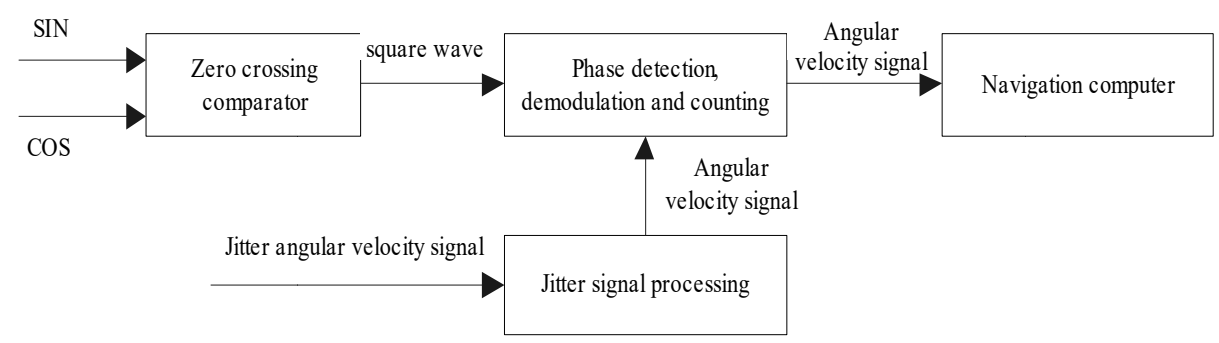

Fig 11. Block diagram of the whole cycle sampling

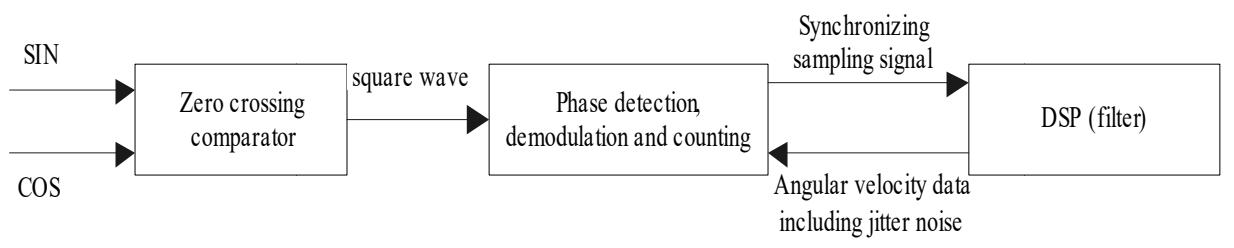

Fig 12. Block diagram of digital filter

The FIR filter adopts 46 order, the low-pass bandwidth of Through MATLAB simulation, we can clearly see the the filter is $200 \mathrm{~Hz}$, and the cut-off frequency is $300 \mathrm{~Hz}$. performance of the filter, as shown in the following figure:

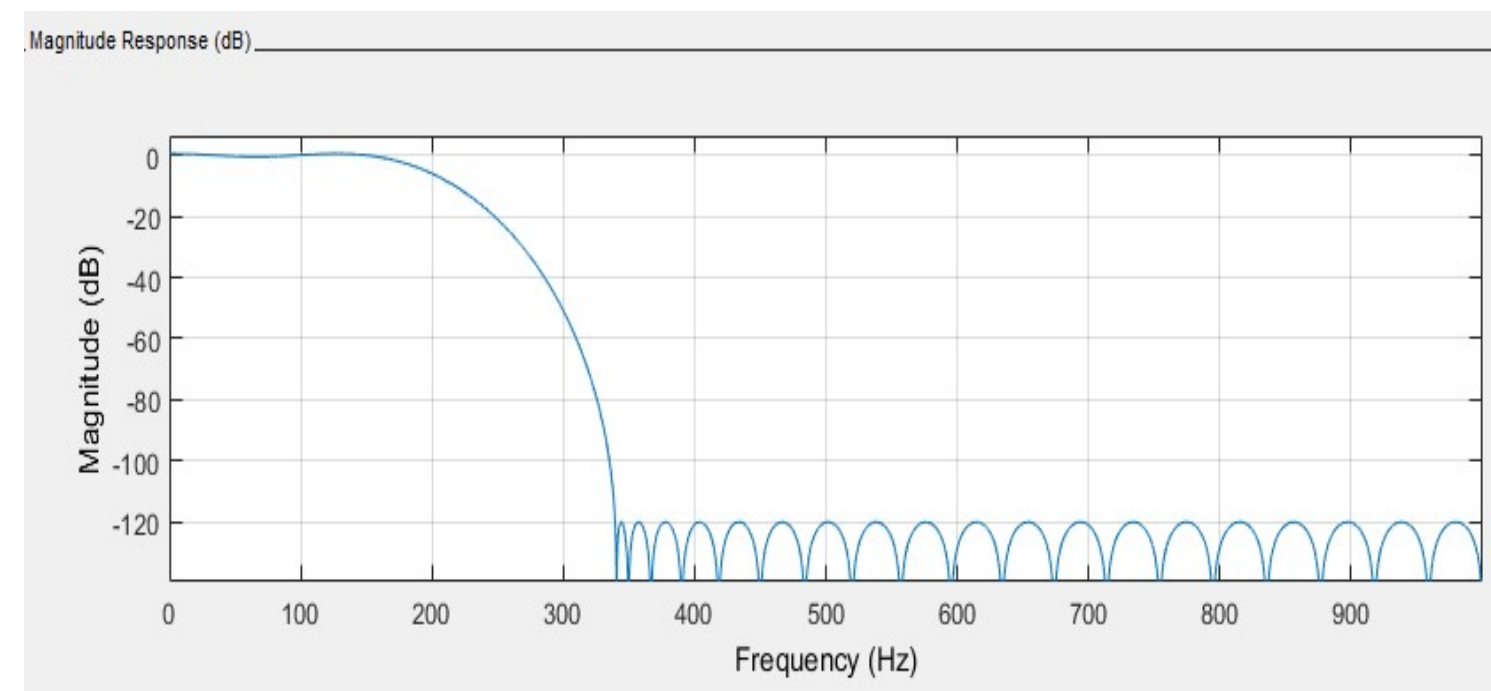

Fig 11. Amplitude frequency characteristic diagram

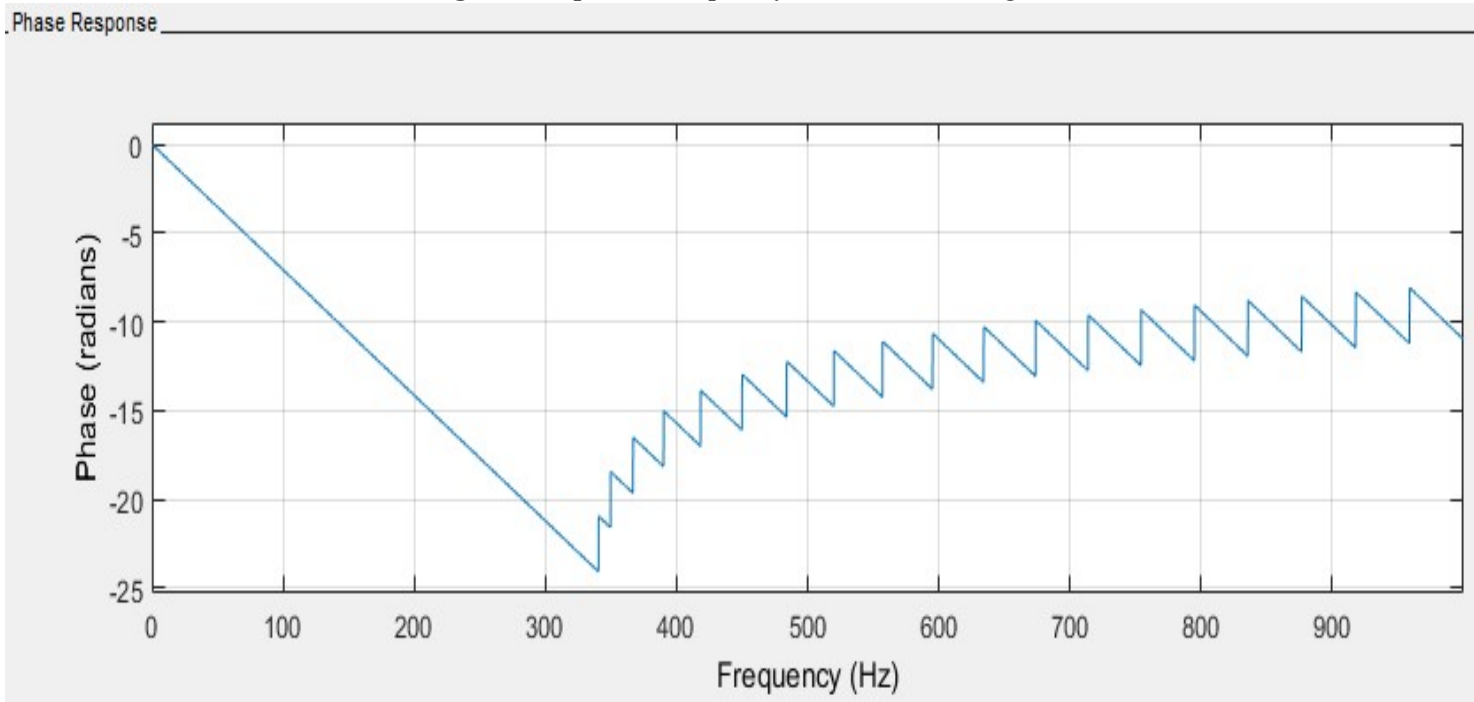

Fig 12. Phase frequency characteristic diagram 


\section{B. Data transmission delay analysis}

After receiving the synchronization signal command from the time system module, the system immediately sends the attitude data to the time system module. At this time, the data sent is the attitude data that has been calculated by the system, and the attitude transmitted to the time system module has a delay. According to the application system settings, the frequency of synchronization signal is set to $100 \mathrm{~Hz}$ in this paper, and the synchronization signal is consistent with the update frequency of attitude data.

The attitude output frequency of Laser Strapdown Navigation System is $400 \mathrm{~Hz}$, i.e. $2.5 \mathrm{~ms}$. In the process of hardware sampling integration, the integration is completed at every $2.5 \mathrm{~ms}$ interval, and the delay time is ignored. Only the navigation calculation and attitude data transmission delay time are considered.

\section{The sampling period is consistent with the synchronous signal}

The laser gyro strapdown inertial navigation system starts sampling after receiving the synchronous signal, and the sampling time is $2.5 \mathrm{~ms}$. When the next synchronization signal is received, the positioning and orientation system starts sampling again, and carries out navigation calculation at the same time, and sends the attitude calculated last time to the time system module. In this case, the attitude transmitted to the time system module has a fixed time delay of $\mathrm{T}=2 \mathrm{~ms}+\Delta \mathrm{T}$, that is, the sampling period of the system $(2.5 \mathrm{~ms})$ plus the time of the system transmitting to the time system module (a small amount of $\triangle T$ ).

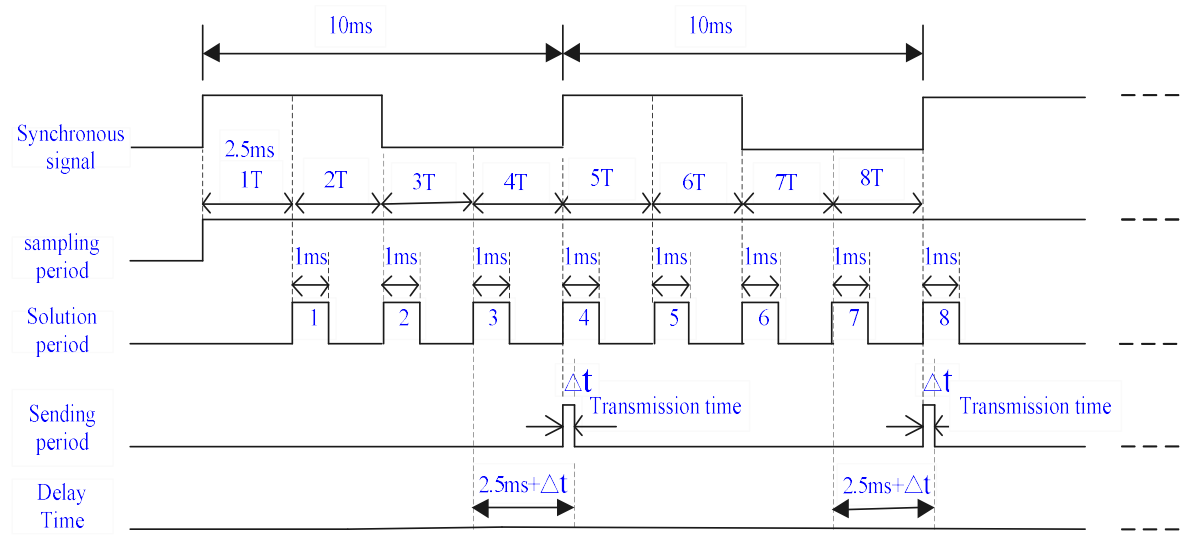

Fig 13. The sampling period is consistent with the synchronous signal

The sampling period is inconsistent with the synchronous signal

When the system receives the synchronous signal, there may be two states in the system state

State 1: the system is sampling but not solving
The attitude delay includes the solution time $(1 \mathrm{~ms})$, the time $\mathrm{T} 1(0<\mathrm{T} 1<1.5 \mathrm{~ms})$ between the received synchronization signal and the solution, and the time $\Delta \mathrm{t}$ (a small amount) for the system to transmit the attitude to the time system module. The total attitude delay time is $\mathrm{t}=1 \mathrm{~ms}+\mathrm{T} 1+\Delta \mathrm{t}$.

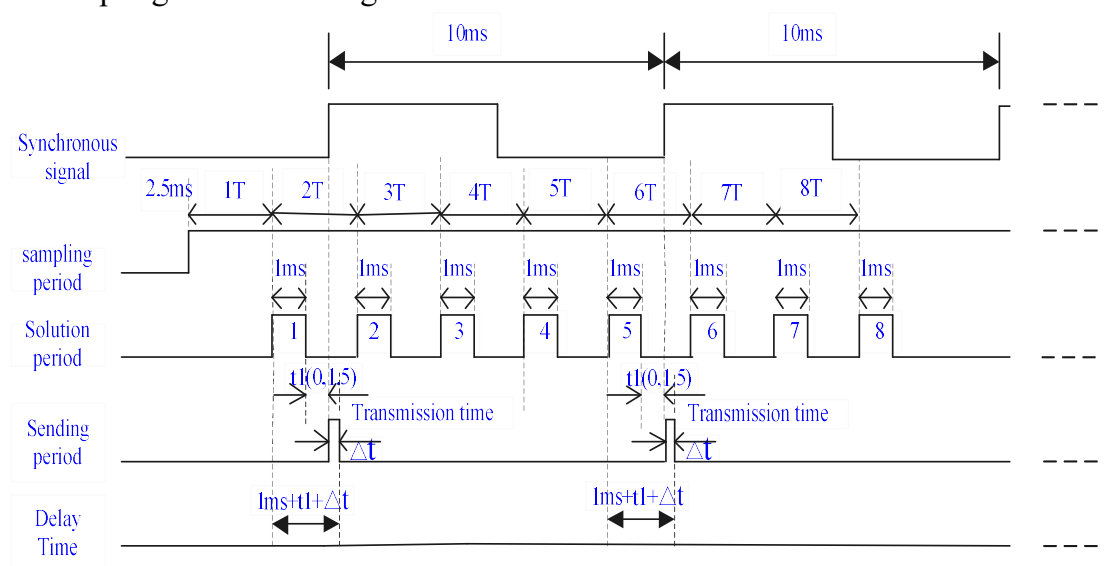

Fig 14. Out of period of synchronous signal time calculation

State 2: the system is sampling and solving the previous cycle

When the system receives the synchronization signal, it is in the process of solving. At this time, the attitude data transmitted to the time system module is the attitude completed in the previous cycle. The attitude delay includes the time of solving the sampling $(2.5 \mathrm{~ms})$, the time $\mathrm{T} 2(0<\mathrm{T} 2<1 \mathrm{~ms})$ when the synchronization signal is received and the time $\Delta \mathrm{t}$ (a small amount) when the system transmits the attitude to the time 
system module. The total attitude delay time is $2.5 \mathrm{~ms}+\mathrm{T} 2+\Delta \mathrm{t}$.

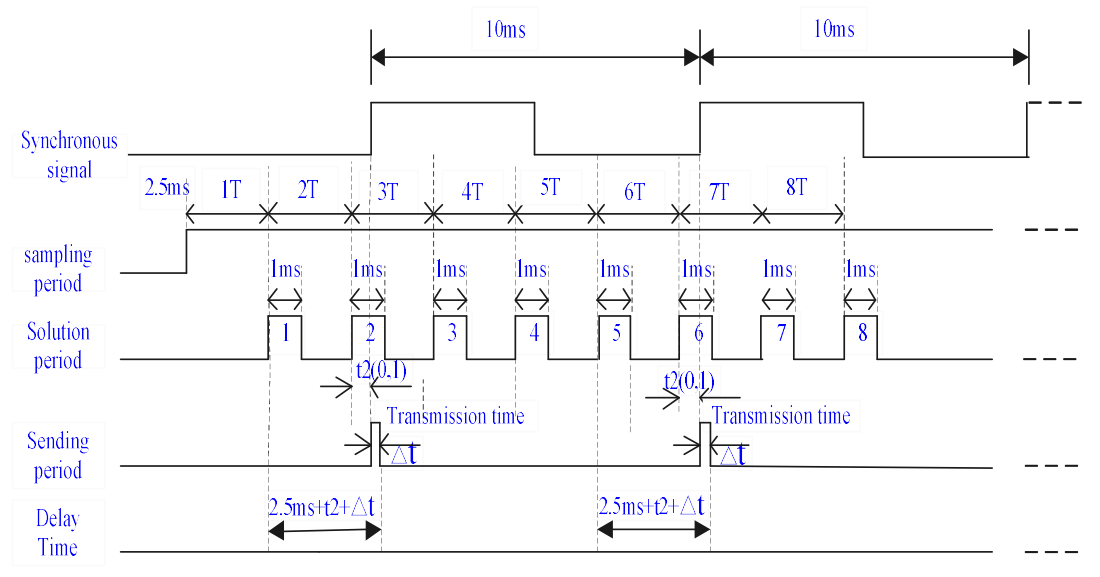

Fig 15. Time calculation period of synchronous signal

\section{CONCLUSION}

Attitude real-time is the difficulty of inertial navigation system application. Whether it can effectively improve the system's rapid response and improve the vehicle's maneuverability, this paper focuses on the inertial components of three-axis laser gyroscope and three-axis accelerometer sensor of strapdown inertial navigation system, according to the real-time problems of signal acquisition, attitude calculation and data output of laser gyroscope and quartz disturbing accelerometer, this paper analyzes the inertia The data input and output characteristics of IMU, odometer, altimeter, GPS / BeiDou and other related components are navigated. The parallel processing of multi CPU, the exchange of RS-232 serial interface and can bus interface are analyzed. It is difficult to meet the problems of high-speed real-time sampling and data input and output data exchange of sensors in motion process. A high-speed data access method using FPGA dual port RAM + Dual DSP is proposed The clock synchronization period can be set according to the system application to realize the real-time navigation data such as heading, roll, pitch, longitude, latitude, elevation, etc., which can effectively solve the bottleneck problem of data transmission, processing and transmission to each equipment. The maximum output frequency attitude rate can reach $2000 \mathrm{kHz}$, which improves the cycle calculation ability of the system and realizes the attitude state of strapdown navigation system Quick response ability.

\section{ACKNOWLEDGEMENTS}

This work was supported by Chongqing higher education teaching reform research project: research and practice of informatization teaching based on blue ink cloud class in management courses (No.: 183263), exploration on Ideological and political teaching reform of performance management course (No.: 193472), research on behavior data application in students' Ideological and Political Education under the background of big data (No.: 19sksz089), intelligent navigation innovation team (No.: cqy2018) TD05).

\section{REFERENCES}

[1] S. P. Zhang, C. Q. LI, and J. F Zhang, "Design of a High-speed Real-time Data Acquisition and Processing System", Fire Control \& Command Contro, vol.41, no.7, pp. 125-129, 2016.

[2] J. Z. Wang, "Error Calibration of IMU in Laser Gyro Strapdown Insertial Navigation System", Piezoelectrics \& Acoustooptics, vol.40, no.3, pp.448-453, 2018.

[3] B. Pang, Q. Li. "Multi-Channel Strap-Down Inertial Navigation of Data Acquisition System", Computer Systems \& Applications, vol.23, no.3,pp. 195-198, 2014.

[4] B. Tang, J. M. Li, S. Z. LI, "Laser gyroscope signal demodulate system based on FPGA", Measurement Control Technology and Instruments, vol.39, no.12,pp. 1-7, 2018.

[5] B. Xu, L. Z. Wang, W. H. Wu, S. X. Li, T. H. Duan, "SINS alignment method in air based on slant and angle position assisted by radar", Journal of Chinese Inertial Technology, vol.27, no.5,pp. 573-579, 2019.

[6] Z. W. Wangi, J. Qin, G. L Yang, "Initial alignment of GPS/INS moving base with large misalignment angle", Piezo-electrics \& Acoustooptics, vol.27, no.5, pp. 121-129,2018.

[7] M. A. Rahgoshay, P. Karimaghaie, F. Shabaninia, "Robust inertial frame-based alignment of fiber-optic gyro strap-down inertial navigation systems using a generalized proportional-integral-derivative filter", Optical Enginee-ring, vol.56, no.9,pp. 1-10, 2017.

[8] L. Chang, H. Zhang, Z. Z. Gao, H. X. Ding, Q. Zhong, "The Application of Zero Update in GNSS/INS Tightly Coupled Integation”, Journal of Geodeay and Geodynamics, vol.36, no.10, pp. 879-883, 2016.

[9] H. Xu, F. P. Sun, S. Liu, Y. W. Miao, J. Shi. "The Application of Zero-Velocity Update in the GNSS/INSS Integrated Navigation". GNSS World of China, vol.39, no.4, pp. 43-45, 2014.

[10] Y. Y. Xue, C. Liu, Y. Chen, K. L. Xu, M. Wang, Y. C. Yang, "Design and Realization of Inertial Attitude Derermination System Base on OMAPL138 and FGGA", Computer Measurement \& Control, vol.25, no.10, pp. 1-14, 2017.

[11] J. Z. Wang, "Research on Zero Speed Correction of Laser Gyro Strapdown Inertial Navigation System", Piezoelectrics \& Acoustooptics, vol.40, no.4, pp. 426-432, 2018.

Jianzhong Wang was born in Pengan, Sichuan Province, in 1979. He is a senior engineer with double master's degree. Currently, he is a professor in College of Artificial Intelligence of Chongqing Youth Vocational and Technical College, China. His major research interests include computer information technology and inertial navigation.

Yan Zhang was born in Jingzhou, Hubei Province, in 1987. She is a Master of management. Currently, she is a instructor in department of Economics Management of Chongqing Youth Vocational and Technical College, China. Her major research interests include business management and Human Resource Management. 
Jijun Yan was born in Yongchuan, Chongqing, in 1976. He is a senior lecturer, graduate student, master of management engineering and science, he is a instructor in department of Economics Management of Chongqing Youth

Vocational and Technical College, China. His major research interests include big data and statistics research.

\section{Creative Commons Attribution License 4.0} (Attribution 4.0 International, CC BY 4.0)

This article is published under the terms of the Creative Commons Attribution License 4.0

https://creativecommons.org/licenses/by/4.0/deed.en US 